\title{
Germanica
}

\section{Le fantastique comme symptôme et poétique dans Die Weissagung d'Arthur Schnitzler}

Das Phantastische als Symptom und poetjsches Mittel in Die Weissagung von Arthur Schnitzler

\section{Geneviève Rousse}

\section{OpenEdition}

Journals

Édition électronique

URL : http://journals.openedition.org/germanica/2829

DOI : 10.4000/germanica.2829

ISSN : 2107-0784

\section{Éditeur}

Université de Lille

\section{Édition imprimée}

Date de publication : 30 juin 1988

Pagination : 185 à 203

ISSN : 0984-2632

Référence électronique

Geneviève Roussel, « Le fantastique comme symptôme et poétique dans Die Weissagung d'Arthur Schnitzler », Germanica [En ligne], 3 | 1988, mis en ligne le 11 septembre 2015, consulté le 06 octobre 2020. URL : http://journals.openedition.org/germanica/2829 ; DOI : https://doi.org/10.4000/ germanica.2829

Ce document a été généré automatiquement le 6 octobre 2020.

(c) Tous droits réservés 


\title{
Le fantastique comme symptôme et poétique dans Die Weissagung d'Arthur Schnitzler
}

\author{
Das Phantastische als Symptom und poetjsches Mittel in Die Weissagung von \\ Arthur Schnitzler
}

Geneviève Roussel

Schnitzlers Erzählung, Die Weissagung und

ähnliche

mit dem Wunderbaren liebäugelnde

Produktione...

S. Freud

L'admiration ambivalente de Freud pour Schnitzler, son étonnement respectueux devant son double en littérature, sont connus. Freud, dans son étude sur l'étrange, laisse même poindre la jalousie du clinicien qu'entrave la réalité, quand il évoque les libertés et privilèges de !'écrivain, que la fiction dote de tous les moyens de l'esthétique. Le voici accusé de traîtrise, cet écrivain contemporain qui, ne faisant pas (ou plus) usage des repères classiques - démons et autres fantômes -, n'introduirait le fantastique dans une fiction d'apparence réaliste qu'en catimini, à la déloyale. Et Schnitzler... plus précisément son récit $L a$ prédiction ${ }^{1}$ est tenu pour exemplaire de ces productions décevantes aux moyens douteux, aux effets impurs. Freud, lecteur abusé, sanctionne, par la colère et le ressentiment, la tromperie : le flirt avec le fantastique ${ }^{2}$.

Er verrät uns [...], er betrügt uns, indem er uns die gemeine Wirklichkeit verspricht und dann doch über diese hinausgeht. Wir reagieren auf seine Fiktionen so, wie wir auf eigene Erlebnisse reagiert hätten; wenn wir den Betrug merken, ist es zu spät, der Dichter hat seine Absicht bereits erreicht, aber ich muss behaupten, er hat keine reine Wirkung erreicht. Bei uns bleibt ein Gefühl von unbefriedigung, eine Art von Groil über die versuchte Täuschung, wie ich es besonders deutlich nach der Lektüre von Schnitzlers Erzählung Die Weissagung und ähnlichen mit dem Wunderbaren liebäugelnden Produktionen verspürt habe. 
2 Flirt avec l'inquiétante étrangeté, marges du fantastique? Dès la parution de la nouvelle dans le recueil de 1907 La Pénombre des Âmes ${ }^{3}$, une alléchante prière d'insérer avait noté la parenté avec Poe. Schnitzler, dans une lettre indignée à son éditeur ${ }^{4}$, protesta contre ce texte publié à son insu et s'éleva contre une lecture de ses nouvelles comme " variations sur des thèmes de l'univers d'Edgar Poe » : " analyse superficielle ", " étrange remarque », même pour La prédiction, affirma-t-il. Souci de se démarquer de toute une production à la mode, même sous l'égide de Poe? Ou refus du fantastique? Dès les années 80-90 Schnitzler s'intéressa pourtant, lui aussi, à l'occultisme, à la magie. Bien plus, médecin, assistant en neurologie pathologique, expert en hypnotisme à des fins cliniques, il se livra, ès qualités, à des expériences scientifiques sur les problèmes d'hypnose, médiumnité, suggestion, magnétisme, qui débouchèrent d'ailleurs sur la publication d'une étude ${ }^{5}$, et, toujours dans le cadre médical, à des expériences qu'il qualifia de psychologiques (faire vivre à des médiums en état d'hypnose toutes sortes de situations et actions pouvant aller jusqu'au meurtre $\left.!^{6}\right)$; il les abandonna en constatant qu'elles sont dommageables pour les médiums, tant pour leur santé que pour l'équilibre de leur personnalité.

Les œuvres de cette époque, Paracelse, Au Perroquet Vert, La prédiction..., portent, à divers titres, des traces de ces expériences, qui contribuent à nuancer l'analyse de Schnitzler sur les rapports entre apparence et réalité.

... das rätselhafte Abenteuer, das sich löst, ohne sich aufzuklären.

La prédiction est présentée comme le récit (Bericht) rédigé, à des fins plus ou moins scientifiques, par un écrivain depuis lors disparu, pour relater le 9 septembre 1868 tel qu'il l'a vécu. Ayant écrit une pièce pour la troupe d'amateurs d'un baron passionné de théâtre, l'écrivain vient assister à la représentation qui va en être donnée ce soir-là dans la propriété de cet aristocrate. Dans l'après-midi il reçoit la visite du neveu du baron, Franz von Umprecht. À la suite de désistements, Umprecht interprétera le rôle principal. Il fait à l'écrivain le récit de l'étrange aventure qui depuis dix ans régit sa vie. Dix ans plus tôt en effet, jour pour jour, par un soir d'ennui dans une garnison de Pologne, un ce1tain Marco Polo, magicien, était venu divertir les officiers. Il lut dans la main du commandant sa mort prochaine. Umprecht pour sa part, le pria de lui montrer un instant de sa vie future, «dans dix ans par exemple » : dans l'image surgie, il s'est vu lui-même, au milieu d'une clairière plongée dans la nuit, allongé sur une civière, une cicatrice au front, entouré d'une belle femme rousse et d'enfants en pleurs. Le commandant étant mort quinze jours plus tard, Umprecht, sur les conseils d'un ami poète, confia aux scellés d'un notaire le schéma de sa vision, qui ne lui serait restitué que dix ans plus tard. Ayant quitté l'état militaire, il a dès lors évité tout ce qui aurait pu ressembler à un début d'accomplissement de la vision : domaines avec clairières, femme rousse, enfants, et même duel qui entraînerait cicatrice. Pourtant il a cru évidemment ! - reconnaître l'acharnement du destin : son domaine, celui de son oncle comportent des clairières, sa femme s'est teint les cheveux en roux, à son fils unique est venue se joindre une nièce orpheline, un accident de train lui a laissé une cicatrice au front l'Invité pour l'été $68 \mathrm{chez}$ son oncle, il prévoyait de fuir au bord de la mer avant la date fatidique... Pourtant, en ce jour du 9 septembre, il répète, chez son oncle, comme il le fait depuis quelques semaines, la scène de sa mort - théâtrale. Car la pièce proposée au baron par l'écrivain se termine par l'exacte scène de sa vision, et la représentation 
dénouera, pense-t-il, le maléfice : il vient remercier l'écrivain et lui présente, pour preuve de son récit, le schéma scellé déposé chez le notaire.

5 L'écrivain y reconnaît sa dernière scène : décors, situation, personnages. Y figure même, à son grand étonnement, un personnage annexe, initialement prévu, vieil homme chauve à écharpe verte, auquel il a renoncé finalement. Troublé par la relation qui semble exister entre Umprecht et lui-même, l'écrivain assiste à la représentation : au dénouement son héros, de retour d'aventures, doit mourir en scène. Umprecht joue l'agonisant. Un vieil homme chauve à écharpe verte traverse la scène à la poursuite de sa perruque et disparaît. Le public croit que la pièce se poursuit. L'écrivain, voyant l'acteur ne pas se relever, sait que Umprecht vient de mourir en scène. Horrifié il quitte le domaine. Quand, le lendemain, il fait part de toute l'histoire à l'oncle du mort et apporte pour preuve le schéma notarié, la feuille est vierge... Le vieil homme à écharpe verte, un maitre d'école du voisinage, ne sera jamais retrouvé, ni vif, ni mort.

6 Au fil de la nouvelle le lecteur aura vu surgir énigmes, contradictions, coïncidences explications transitoires qui répercutent l'inquiétude -, tendues vers un dénouement attendu comme logique bien qu'improbable. L'écriture, la logique narrative, les motifs et leurs connotations semblent faire de la nouvelle de Schnitzler un récit fantastique exemplaire! Mais la mort, finalement réelle, non seulement rêvée ou jouée, est-elle la mort pré-dite? Ou bien la mort pré-vue, par un individu en quête d'avenir, c'est-à-dire voulue, et partant réalisée?

7 La littérature fantastique du XIXe siècle serait, affirme Todorov, la mauvaise conscience de ce siècle positiviste, la remise en question de sa «métaphysique du réel et de l'imaginaire ${ }^{7}$ ». En 1900 l'opposition entre réel et irréel n'est plus irréductible. L'ambiguïté naît certes de l'interrogation sur les parts respectives du réel et de l'imaginaire (revu et corrigé en subconscient, inconscient, etc...), mais plus encore de l'interrogation sur leur interaction. L'incertitude naît, dans la nouvelle de Schnitzler, de la course parallèle de deux probabilités externes. La lecture méta-empirique suivra le cheminement de l'extra-naturel, ici représenté par la prédiction, le mauvais sort jeté par le magicien, réalisé par le destin, parachevé par l'écrivain, instrument de ce destin. La lecture empirique reconnaîtra la pathologie du délire morbide. Le lecteur naïf, épris d'intrigue, fera d'abord la lecture méta-empirique, celle que Schnitzler qualifie de "superficielle ", mais qu'il sollicite en usant des recettes du conteur fantastique.

La prédiction est racontée comme une nouvelle fantastique. Un événement anormal vient déranger l'ordre d'un univers jusqu'alors structuré: les "prédictions » du magicien, que l'officier analyse comme simples artifices, jeux de miroirs par exemple, jusqu'à ce que la mort subite du commandant leur confère une menaçante vraisemblance. Les périls qui s'accumulent ensuite rendent inégal le combat avec le sort. Lecteurs, nous partageons la peur du héros devant l'accumulation des coïncidences, ses efforts pour les expliquer, leur échapper. Quand il pense gagner la partie, son soulagement trouble l'auteur de la pièce, étonne le lecteur, impatient de vérifier que la pointe sera bien - le piège du fantastique a fonctionné - la mort du héros.

9 L'impression de cohérence interne de l'univers ambigu de la nouvelle, ce que Barthes appelle l'effet de réel, est obtenue en particulier par l'assimilation entre le héros Umprecht - et le narrateur - auteur de la pièce -, qui fait sien le récit du héros («Sie konnen nicht wissen, wie meine Wortegemdnt sind. Darlich Sie bitten, mich anzuhören?» Mit offenbara Absichtlichkeit sa ruhig ais moglich scheinend, begann er : «Ich bin»...). Le narrateur écoute d'abord, par simple politesse, le monologue de son interlocuteur, qui détaille les 
circonstances de la vision et la vision puis, symétriquement, les prémisses de sa réalisation. Ensuite, devant le plan notarié, preuve de leur inexplicable relation, « un plan complet, comme conçu par moi-même", dit le narrateur, un bref dialogue (ich fragte, ... er sagte, ... ich nehme an, ... wie sagten Sie ?) noue définitivement leur complicité (wir sch wiegen eine Weile, ... wir wurden unterbrochen) : ils vivent dès lors une seule et même aventure.

10 Le narrateur, impliqué comme écrivain, co-auteur réaliste de la scène fantastique, adopte la subjectivité du héros (Und so unwahrscheinlich, ja so ungeheuerlich sein ganzer Bericht mir erschien - irgend etwas in mir verlangle sogar danach, ihm glauben zu dürfen). Narrateur-témoin, il cautionne l'exactitude du dénouement - sa conformité avec la prédiction - et rétrospectivement le monologue de Franz von Umprecht.

11 L'autorité du narrateur est interne à la fiction. Schnitzler lui superpose une autorité historique en ancrant sa nouvelle dans un univers précis, familier au lecteur de 1900, typique de la société autrichienne des années 60 , authentifié par les références géographiques (on pourrait situer la propriété du baron sur une carte d'état-major), mondaines (le baron Meudolt, le notaire Artimer, le prince du régi ment), culturelles (le poète Friedrich von Gulant, le peintre danois Petersen). En outre Schnitzler lui-même prend le relais, intervient, par l'intermédiaire d'une « postface de l'éditeur », pour une sorte de coordination scientifique de l'ensemble. L'écrivain, rédacteur du récit, était, nous apprend-t-il, l'ami d'un médecin de Merano, qui lui avait fait faire la connaissance du baron. Ayant hérité par la suite des papiers de l'écrivain, ce médecin transmet, une dizaine d'années plus tard, le manuscrit du récit à l'éditeur, à l'occasion d'une discussion entre eux sur esprits, télépathie et présages. Il ne peut s'agit d'une fiction (eine frei erfundene Erzählung), puisque le médecin de Merano a assisté à la représentation théâtrale, vu mourir Umprecht et connu en outre le vieil homme à écharpe verte mystérieusement disparu. L'éditeur ajoute que, pour sa part, il se souvient avoir lu le nom du magicien Marco Polo, sur une affiche en province, dans sa jeunesse.

12 Schnitzler parfait l'ambiguïté de son texte en minant l'authentification du récit par le récit lui-même, truffé de procédés créateurs d'hésitation. Dans son monologue, Umprecht, s'imposant le recul d'un récit au passé, semble prendre ses distances par rapport à l'insolite. Il souligne le côté étrange de son aventure (unbegreiflich - rätselhaft - geheimnisvoll - sonderbar - seltsam - drohend - eigentümlich). Il use d'un langage oblique, chargé des locutions propres à la modalisation (Ich versichere Sie, uns allen war, als ab der Oberst gezeichnet worden wär, ... von Jahr zu Jahr, als triebe ein launisches Schicksal sein Spiel mit mir, ... Wiesenpartie, die mir in eigentümlicher Art der Örtlichkeit zu gleichen schien, vor der mich zu hüten ich vielleicht allen Anlass hatte, ... ich glaubte eine Ähnlichkeit mil den Zügen des Knaben aus dem Traum zu entdecken; bald schien sie sich zu verwischen, ... ich kam mir vor wie in sicherer Hut ...).

13 Schnitzler, enfin, ne néglige pas un usage discret d'éléments conventionnels du fantastique, créateurs d'ambiance. Ainsi retrouve-t-on des jeux d'ombre et de lumière, une femme rousse, un héros marqué au front du sceau de l'élection, un magicien juif en uniforme satanique : brocart rouge, redingote noire, haut-de-forme et lunettes contre le soleil! Ces motifs porteurs de l'étrange sont par ailleurs chargés de tisser un réseau de signes interne à la nouvelle.

Le thème du regard, cher à Hoffmann, est présent dès l'entrée en scène du magicien aux yeux fragiles mais perçants. Marco Polo maitrise mal la parole, mais manie 
parfaitement l'arme du regard (Mein Freund Marco Polo hat scharfe Augen). Il lit la mort du commandant dans les lignes de sa main, après lui avoir jeté un regard plein de mépris. Pour mieux voir, il s'éloigne des lumières du salon (Hier sieht man schlecht), recherche le clair de lune. Son talent est de regarder (Marco Polo sah mich an, ... Marco Polo sah mich starr an), son don de faire voir (Irgend einen Augenblick aus Ihrem künftigen Leben könnte ich Ihnen zeigen wie ein Bild).

Apparenté au thème du regard, le thème du double surgit dans le récit de Umprecht au moment décisif où le destin le marque au front (der Augenblick, der mich am tiefslen zum Bewusstsein meiner Wehrlosigkeit brachte): dans un train une pierre lancée à travers la fenêtre le blesse au front. Les voyageurs s'interrogent : attentat, plaisanterie ? Seul un homme se tait, comme enfoncé dans son coin, immobile et le regard fixe. Sur le quai de la gare, surgi soudain aux côtés du blessé, il prononce une phrase : «c'est moi qui étais visé »..., puis disparaît. Il ne sera pas possible de le retrouver. Umprecht suggère que l'homme souffrait peut-être de la "maladie de la persécution», et pose ainsi le diagnostic de sa propre maladie!

Dualité de l'argument - naturel/ surnaturel -, dualité du récit - subjectif / objectif renvoient à l'un des paradoxes premiers du fantastique, celui de la mort dans la vie, de la vie dans la mort. Freud aurait-il, devant la nouvelle de Schnitzler comme devant L'Homme au sable de Hoffmann, refusés d'admettre ce paradoxe, d'accepter le non-sens du fantastique, et de regarder monter de la lecture le fantôme de sa propre peur?

... als triebe ein launisches Schicksal sein Spiel mit

mir.

17 La prédiction est l'une des nouvelles du recueil La Pénombre des Âmes (Dämmerseelen). Les personnages principaux de ces nouvelles ${ }^{9}$ ont en commun d'être des âmes crépusculaires, caractérisées par une sorte de faiblesse, d'absence à la vie. Il ne s'agit pas de décadents fin-de-siècle, esthètes d'un mal de vivre nourri de luxe, auxquels on a trop souvent réduit les personnages de Schnitzler - La prédiction par exemple se passe entre 1858 et 1868 -, mais d'individus de tous milieux, hommes ou femmes, qui ne maitrisent pas leur vie; faute d'accord avec eux-mêmes ou avec le monde, ils apparaissent comme déracinés, solitaires. Cet isolement entraîne une sorte d'effritement de leur personnalité, et dans les failles du moi peuvent se glisser tous les germes du pathologique : suicides, morts subites, folie sont les échappatoires ultimes.

18 Le héros de La prédiction n'est à première vue pas particulièrement fragile : à peine quarante ans, formé à la discipline militaire, ancré dans un système de références morales, installé dans une vie familiale et sociale conforme à son rang et à ses vœux. Pourtant ses premiers mots sont révélateurs : il vit, dit-il, depuis dix ans "pour ainsi dire dans l'ombre d'une incompréhensible aventure », la prédiction de Marco Polo. Il en détaille les circonstances afin de démontrer que, dans un environnement déstabilisant, il était resté parfaitement lucide et digne. En une demi-page il brosse de la vie de garnison - ici un trou à la frontière polonaise - une image plus négative encore que celles de Roth ou Buzzati. Le service, pas même assez astreignant pour apaiser les corps, est un "passe-temps" au même titre que le jeu ou la boisson. Aucune perspective de mutation. Les militaires supportent mal cette vie sinistre (Nicht alle von uns verstanden es ein Leben in dieser trostlosen Aussicht mit Fassung zu tragen). L'un se suicide. Un autre se met à boire, perd la tête, cherche querelles, est limogé. Un capitaine jette sa femme par la fenêtre : elle s'en sort indemne (rätselhafterweise, précise Umprecht), il meurt à l'asile d'aliénés. Un jeune cadet particulièrement sot fait une 
fixation philosophique sur Kant et Hegel, qu'il apprend par cœur. Umprecht se résigne à s'ennuyer "monstrueusement", jusqu'à par moments craindre de devenir fou (ichfürchtete verrückt zu werden).

19 Dans un présent d'ennui, devant un futur sans avenir, les officiers, après la prédiction de mort faite au commandant par Marco Polo, affectent un manque de curiosité. Umprecht seul, discrètement, ordonne au magicien de lui dévoiler son avenir (Prophezeien Sie mir), de lui montrer une image de sa vie future. Après «l'apparition » il s'efforce tout d'abord de croire qu'elle a été « produite » par quelque trucage. À la mort du commandant, il ressent pourtant « une certaine inquiétude ».

Certes, il serait temps encore pour lui de se libérer de la sorcellerie. Exactement comme il a, le soir des prédictions, délivré de l'hypnose le « cadet philosophique » que Marco Polo avait crucifié sur le mur, l'a ramené à la réalité, en le prenant par le bras. Mais en exigeant une prédiction, Umprecht a noué une sorte de pacte diabolique. L'image imposée par le juif lui tiendra lieu de destin, ce destin qu'il semble peu apte à se forger lui-même. Incapable de rompre le pacte - en l'ignorant -, il s'enfonce dans une angoisse de vivre, dont seule la mort pourra le délivrer. Peur de vivre, peur de mourir, plaisir pris à ces peurs sont autant de formes de narcissisme et d'impuissance.

21 La prédiction devient une raison d'être et une forme de vie que Umprecht qualifie avec des termes qui lui sont familiers : le jeu, le combat, avec, puis contre le destin. Il réduit tout d'abord la prophétie à une " histoire ridicule ", puisque, dit-il, il a son « destin bien en mains» et que "rien au monde ne peut le contraindre» à se conformer, le 9 septembre 1868, à une simple vision. Mais l'énoncé de tout ce qu'il décide d'éviter est le catalogue des obsessions qui dorénavant domineront sa vie. Même réduite à une " vision ", puis minimisée en "rêve " (jener Traum, wie ich den Inhalt jener Erscheinung bei mir zu nennen pflegte), la prophétie « empoisonne » sa vie.

Umprecht collectionne les éléments de sa vision tout en se rassurant avec l'idée que, lorsqu'il croit les reconnaître, ce sont peut-être des souvenirs qui viennent se plaquer sur la réalité ! Mais il n'en continue pas moins à comptabiliser les « mises en garde du destin »! La dangereuse femme rousse, par exemple... Sa femme, en son absence, s'est fait teindre les cheveux - parce qu'il avait semblé subjugué dans un musée par le portrait d'une femme rousse. Il croit reconnaître la femme de sa vision: "sa ressemblance avec la femme du rêve - je n'avais pas vu son visage - me sembla totale »! Il envisage, "une idée frisant la folie », de se séparer de sa femme et des enfants, ce qui mystifierait le destin (ich hätte das Schicksal zum Narren gehalten). Une teinture fera l'affaire. Mais à l'occasion d'un duel, le destin à son tour pourrait bien récupérer un «nouvel atout». Umprecht préfère se soustraire au duel, malgré la pression sociale, - Schnitzler souligne qu'il rougit en avouant cette -, pour éviter la cicatrice sur le front, dont il a "une peur panique ». Elle lui est pourtant infligée dans un accident de chemin de fer. Umprecht voit là la preuve que la vie est « un combat inégal avec une force qui (le) nargue ", et il attend l'issue fatale.

Mais en lisant le dénouement de la prière qui doit être jouée à la date fatidique, Umprecht y trouve trait pour trait la situation de la prophétie. Ainsi il suffirait de mimer la mort pour lui échapper. La vie redevient simple et facile. Mais en se déclarant rassuré, Umprecht avoue qu'il n'est pas délivré de l'obsession : il a, dit-il, « l'impression d'être comme en sécurité ", et il serait "à nouveau livré à cette puissance inconnue ", s'il ne jouait pas le rôle. 
24 Au moment même où il se croit sauvé, Umprecht, comme bon nombre des héros de Schnitzler, est perdu. La vision de 58 était la manifestation voilée de son désir de mort ; la réalisation fictive de ce désir - jouer la mort de son double, l'acteur - coïncide avec la réalisation effective. La névrose auto-destructrice du héros, - ce que Freud appellera la «toute-puissance des pensées »-, a consumé son énergie vitale. A l'irruption du joueur de flûte à l'écharpe verte (vert, couleur de Lucifer, du blason des fous, couleur maléfique pour les gens de théâtre), il meurt de saisissement, impuissant à de vivre davantage : « il veut parler - visiblement il en est incapable - il s'effondre ».

La peinture d'êtres crépusculaires, anti-héros par avance, la description du vide d'un certain style de vie sont éminemment subversives. Le drame individuel de Umprecht s'inscrit dans une réalité qu'il ne supporte pas. Après le tournant de 1848, qui met à l'écart une forme d'absolutisme d'ancien régime, les années 60 marquent en Autriche l'essor d'une bourgeoisie montante. L'idéologie du libéralisme triomphe peu à peu. Années de flottement dans l'ordre du monde familier à la petite noblesse, d'où sont issus Franz von Umprecht et son oncle. La vie militaire, autrefois vie de service, est réduite à la dimension de vie de fonctionnaire, que l'on abandonne pour celle de gentilhomme campagnard. Umprecht ne peut assimiler les valeurs de la bourgeoisie, ni s'identifier à celles de la haute aristocratie, qui, par exemple, fraternise avec les juifs, comme on voit le faire un prince, major du régiment, amical avec Marco Polo et poli avec tous les juifs du village, alors que les officiers se comportent systématiquement de manière injurieuse avec eux. Umprecht n'a pas de racines, pas de références paternelles. L'armée n'a été qu'un cadre de vie, où l'Empereur était absent. Pas de château ancestral où se ressourcer. Son oncle cultive, en dilettante, le théâtre, sa tante la vie de salon. Sa femme, trop peureuse et superstitieuse, ne saurait être sa confidente. Coupé de la société nouvelle, Umprecht fuit hors du temps, de l'histoire, dans un autre ordre, celui de l'imaginaire, s'y installe et en meurt.

Worte lassen sich immer in verschiedener Weise auslegen.

L'événement qui rompt l'équilibre apparent d'un quotidien mortellement ennuyeux, fait basculer Umprecht dans sa stratégie de névrose, est la prédiction de Marco Polo. Elle ne lui est pas imposée, pas plus qu'au commandant le présage de sa mort. Il la sollicite, décide la forme que doit lui donner le magicien. Il néglige la classique lecture de l'avenir dans les lignes de la main. "Voilà. Je souhaite savoir quelque chose de plus précis. Les mots se prêtent toujours à des interprétations diverses. Je voudrais savoir ce qui m'arrivera plus tard, disons dans dix ans par exemple». Marco Polo proposant de lui «montrer un instant de (sa) vie future comme une image», Umprecht précise : «Bien, alors je veux voir ce qui m'arrivera dans dix ans ». Une vision prend alors la place du monde des choses - la caserne qu'il « voyait » à l'instant sous la lumière de la lune - et Umprecht raconte qu'il «[s']est vu lui-même, comme on se voit parfois en rêve... [s']est vu vieilli de dix ans, avec une barbe brune, une cicatrice au front, étendu sur une civière "... Militaire, Umprecht se méfie des mots; il assimile par contre instinctivement voir et savoir. Une image, irréelle de surcroît, - suggérée ou autosuggérée - lui tiendra lieu de futurologie.

La nouvelle, que sous-tend l'interaction du mot et de l'image, a donc un titre emblématique. En passant du titre initialement prévu Sorcellerie ${ }^{10}$ au titre. La prédiction, tous deux porteurs, apparemment, de la même connotation fantastique, Schnitzler inscrit son récit dans une autre perspective. L'ambiguïté du fantastique renvoie à 
l'ambiguïté de l'art, à la duplicité de l'artiste, de l'écrivain en particulier. Sans doute toute littérature fantastique est-elle, in fine, interrogation sur le réel et l'irréel, deux univers dont l'écrivain l'écrivain est un traditionnel médiateur, chargé dans le texte du coup de projecteur esthétique ou philosophique sur le fantastique. Dans la nouvelle de Schnitzler il est bien autre chose. Narrateur témoin, il cautionne la présence du fantastique. Auteur, il en réalise une forme sensible - sa pièce, texte et mise en scène -, il l'authentifie. Agent exécuteur du fantastique, il se fait meurtrier par l'écriture, quand la double fiction, fantastique et esthétique, engendre la mort.

La vision insolite initiale prend peu à peu l'épaisseur des mots et devient alors seulement pré-diction, savoir dit. Par trois fois «l'apparition» devient texte : dans le schéma notarié, dans la pièce de théâtre, dans le récit de Franz von Umprecht. Le premier texte, le schéma notarié, est "esquissé » - comme un croquis de metteur en scène, avec indications succintes -, peu de temps après la vision, par un ami de Umprecht, le poète Friedrich von Gulant (Der war es, der mit mir zusammen das Schema entwarj). Umprecht dit avoir alors été " contrarié » par le sérieux du poète, mais avoir ensuite "porté moins d'attention à la chose", en perdant de vue son ami et en apprenant sa mort. Le deuxième texte est le texte théâtral. Umprecht, lisant la pièce proposée à son oncle, reconnaît dans la scène finale «mot pour mot la situation qui (lui) avait été prophétisée pour le neuf septembre »: le mot s'est substitué à l'image. Le troisième texte, le monologue de Umprecht, entretient l'équivoque: l'adhésion progressive à l'univers prophétique se cristallise en adhésion immédiate à l'univers théâtral. Le désordre de la vision, rencontrant le texte, se dénoue dans l'ordre de l'écrit, l'art conjure le surnaturel (... von nun an nahm alles seinen einfachen und guten Gang). Umprecht, à la lumière de la pièce, réorganise sa vie a posteriori : il agence les événements en coöncidences, les années en esquisses de la mise en scène finale. La lecture de la pièce a revivifié et remodelé la vision. Le début de l'aventure fantastique et son accomplissement dans deux espaces fictifs similaires coïncident donc parfaitement, abolissant le temps. Le simulacre de mort doit à la fois exorciser la névrose et participer de l'œuvre d'art : les deux fictions, reflets l'une de l'autre, s'entredétruisent.

29 L'inquiétante étrangeté ne serait donc pas tant celle de la prophétie du magicien, suffisamment justifiée par l'impressionnabilité du héros et son inconscient désir de non-vie -, que celle de la pièce de théâtre, de sa similitude avec la vision, telle qu'elle a été consignée dix ans plus tôt. Le thème du double nourrit la problématique de l'écriture : si la scène finale est une duplication de la vision, l'écrivain est un double du héros.

Umprecht, du reste, remerciant l'écrivain d'avoir écrit «comme pour (lui)» le rôle principal de sa pièce, et de «mettre ainsi fin sans le savoir ni le vouloir à (s)on inexplicable aventure ", ne manifeste pas d'étonnement. Il constate simplement qu'il existe entre eux « une relation démoniaque ».

Das Blatt war weiss, unbeschrieben, unbezeichnet.

31 Dans son diagramme consacré à l'Esprit dans le Verbe Schnitzler oppose « poète » et « littérateur" (Dichter /Literat). Le "littérateur" participe de l'esprit "satanique ", négatif, stérile ; le "daimon» du poète par contre est positif et créateur, le rapproche des dieux. La "relation démoniaque » (dämonischer Zusammenhang) entre l'écrivain et 
lui-même devrait permettre à Umprecht de dénouer le lien prophétique, de renouer avec la vie, le pacte poétique le libérer du pacte diabolique.

Comme lors du face à face avec Marco Polo la relation est d'abord celle du regard. Lors de leur première rencontre, tout au long d'un repas, l'écrivain remarque que Umprecht, dont il n'a qu'un très vague souvenir (il jouait l'année précédente chez son oncle dans une scène muette le rôle d'un moine attablé devant un jeu d'échecs!), le « regarde très souvent, tantôt avec sympathie, tantôt timidement, et sans jamais lui adresser la parole ». Umprecht "se trouble presque », lorsqu'il lui pose une question. Son oncle répond pour lui, en insistant sur « le talent d'acteur, récemment découvert, de son neveu ». Ce qui fait partir Umprecht d'un rire «bizarre », suivi d'un bref regard lancé à l'écrivain, qui commente: « un regard qui exprimait une sorte de complicité entre nous deux, me sembla-t-il, et que je ne pus absolument pas m'expliquer ».

Ce dialogue par le regard crée d'emblée une connivence entre les deux hommes, les isole au milieu de l'univers mondain. Le récit de la prophétie les unit ensuite autour d'un projet commun. La prophétie ne se réalisera pas dans le réel, mais dans l'imaginaire. Le théatre, divertissement, est en outre purification: exorcisme ou thérapeutique. Le microcosme poétique va prendre la consistance de la vie, le jeu se faire réalité, symbolique il est vrai. L'écrivain, plein "d'un orgueil fou », "se ressent comme exécuteur d'une volonté supérieure "... Mais où est la transmission de pensée ? Où le plagiat? Dans la vision racontée par Umprecht à la lumière de la pièce ? Ou dans la scène théatrale lue par Umprecht à la lumière de la vision? En fait, Umprecht a rencontré son double en la personne de l'écrivain, qui a lui-même Marco Polo le magicien pour double ironique.

La structure de la nouvelle met en relief ce thème de la duplication, par ailleurs omniprésent dans le texte. Cette structure très calculée est à la fois symétrique et circulaire. Le monologue de Umprecht occupe toute la partie centrale, le chapitre Il d'une douzaine de pages. Deux chapitres de quatre pages chacun l'encadrent; leur rigoureuse symétrie est renforcée par l'unité de lieu et de temps; la journée du neuf septembre, le riant domaine du baron. Dans le chapitre I l'écrivain relate sa rencontre avec le baron, les circonstances qui l'amenèrent à écrire sa pièce, le hasard qui va le faire assister à la représentation. Dans le chapitre III il raconte la représentation ellemême, la mort de Umprecht, sa dernière rencontre avec le baron. Ces trois chapitres sont commentés a posteriori, comme objectivement, au présent de l'indicatif, dans la très courte post-face attribuée à l'éditeur.

À l'exacte moitié de la nouvelle se situe le pivot du texte ${ }^{11}$, l'instant où, à la mort du commandant, la prophétie faite à Umprecht va devenir son obsession. Autour de ce pivot s'organisent donc symétriquement l'univers fantastique de Umprecht (chapitre II) et l'univers esthétique de l'écrivain (chapitre I et III), reflets l'un de l'autre. Le miroir est plus sombre du côté fantastique, plus souriant du côté de l'esthète. L'écrivain est le double chanceux - ou naïf - de Umprecht.

Psychologiquement les deux hommes ont des traits communs. L'écrivain, lui non plus, n'a pas de racines. Plus vraiment jeune, sans attaches stables à Vienne, il passe sa vie en périples, sans but précis. Il s'était facilement laissé « convaincre ", l'année précédente, de prolonger son séjour au château. "À son grand étonnement" il avait fini par y passer l'été et l'automne. Écrivain dilettante, il avait vu avec plaisir un «modeste rêve de sa jeunesse" se réaliser: le baron avait fait jouer une de ses petites pièces. Comment, dans ces conditions, repousser « l'idée » (Einfall) que lui expose le maître de 
maison "sans pression aucune » (ohne jede Zudringlichkeit) d'écrire une pièce qui serait adaptée au théâtre de plein air du parc, d'autant plus que cette idée correspond «tout naturellement " (ungezwungen) à un de ses projets littéraires? L'écrivain composa la pièce, qui fut acceptée. Il prétend l'avoir ensuite oubliée, et que seul le hasard - il passe l'été sur le lac de Garde tout proche - lui permet d'assister à la représentation. « Il me semble aujourd'hui ", écrit-il dans le même langage oblique que celui de Umprecht, " que j'avais complètement oublié le petit château et toute son animation ".

Cet écrivain de villégiature ne "crée " pas par «nécessité intérieure ». Littérateur "à l'affût de sujets », il " produit » au gré des sensations, expériences, relations ${ }^{12}$. Sa pièce fait preuve d'une certaine frilosité face à la vie. Un homme saisi par le démon de l'aventure quitte les siens un beau matin, sans explication. Il vit en une seule journée tant d'aventures épouvantables qu'il décide de rentrer. Mais, dernière aventure, il est assassiné sur le seuil : pour les siens son départ et sa mort resteront une insoluble énigme.

Umprecht, impressionné par la vision suggérée par Marco Polo, ou auto-suggérée à l'occasion de la présence du magicien, en fait la référence implicite de sa vie, lui conférant par là-même une existence. De même l'écrivain, impressionné par le récit de Umprecht - et pour cause : il lui raconte sa propre pièce, agrémentée d'un suspense inattendu ! - s'identifie à lui. Quand ce dernier brise les cachets du notaire et pose sa " preuve » sur la table, l'écrivain " voit » le plan de la dernière scène, « comme composé par (lui)-même » - Sur ce plan figure la silhouette d'un vieil homme, personnage secondaire oublié. En décrivant minutieusement le vieil homme de sa vision, Umprecht lui redonne une silhouette. L'écrivain une fonction, en faisant appel à ses souvenirs. Et tous deux précisent alors à l'envi, créateurs jumeaux de la fiction.

Après la vision, Umprecht avait d'abord pensé à un trucage de magicien. L'écrivain, après le récit, contemplant le schéma, songe d'abord à une mystification ourdie de longue date par un Umprecht "menteur fantastique » (Nicht für unmöglich hielt ich es anfangs, daß er zu der Art von phantastischen Lügnern gehörte, dit eine Mystification unter Schwierigkeiten von langer Hand bereiten, um sich interessant zu machen)! Mais la bonne impression laissée par Umprecht - et cette agréable sensation de seconder le destin lève ses doutes. La mort en scène de l'acteur principal de sa pièce n'est donc pas pour lui une coïncidence, un hasard. Mais quand il vent à son tour prouver à l'oncle de Umprecht la véracité de ses dires, le papier qu'il sort de sa poche est vierge - une feuille «blanche, sans un mot, sans un dessin »- Umprecht, magicien et artiste du mot à son tour, a amené, par la seule force de son récit, l'écrivain à « voir » le schéma écrit de leur aventure commune, des mots sur papier blanc qui renvoient à l'image sur ciel noir de la vision. Dans La prédiction le héros maîtrise mal sa vie, l'écrivain ne maîtrise pas davantage son œuvre. Leur " relation démoniaque » les rassemble dans l'impuissance.

Suffit-il d'abandonner ces âmes crépusculaires dans le ghetto du fantastique pour que la réalité et ses lois, dites objectives, triomphent - même si c'est sous la forme de la mort - ? Le lecteur doit-il avouer, comme Freud à la lecture de La prédiction, qu'il a été dupé, puisque contraint de réagir aux événements sans pouvoir prendre la distance nécessaire? «Destin et hasard ne sont absolument jamais antinomiques; ils sont une seule et même chose, et d'autant plus incontestablement identiques que le point de vue d'où nous examinons l'événement est plus élevé ", fait remarquer un aphorisme de Schnitzler ${ }^{13}$. Rassénéré, le lecteur peut-il reprendre pied dans l'irréfutable concret? 
41 Schnitzler semble l'y inviter. Tout le récit rédigé par l'écrivain, témoin et narrateur, est rédigé dans les temps du passé. Mais au dénouement de la pièce - et de la prophétie -, il passe subitement du passé au présent, à l'intérieur même d'une phrase ! «On apporta sur la civière Monsieur de Umprecht en aventurier mourant. Les enfants se précipitèrent. Sa femme entra en scène après eux et, les yeux révulsés d'angoisse, elle s'effondre auprès de celui qu'on vient d'assassiner, qui veut s'exprimer une dernière fois, essaie de se relever, mais - comme prévu dans le rôle - n'y parvient plus ». Pour le lecteur ce soudain passage au présent signifie-t-il réalisation de la prophétie? En fait Schnitzler affine le jeu ambigu de la réalité et de la fiction. C'est seulement après cette première mort feinte, théatrale, qu'apparaît le joueur de flûte : «il est chauve, sa perruque s'est envolée; les bras tendus, l'écharpe verte flottant autour du cou, il se précipite sur la scène ». Et que meurt le héros, toujours au présent de l'indicatif ! « Il veut parler - visiblement il en est incapable - il s'effondre ». Il faudra le constat du médecin, la douleur de la veuve, pour que le public réuni dans le parc comprenne qu'il ne s'agit plus de théâtre : "on sait que Monsieur de Umprecht est mort subitement ", de mort clinique, dite naturelle. Quant au lecteur de Schnitzler, les points suspensifs qui achèvent typographiquement ce récit de mort avec répétition lui ouvrent le champ des commentaires, des interprétations. Cette scène au présent, temps de l'objectivité, est l'enclave de l'incontestable, de l'historique, dans la confession du narrateur, qui retourne ensuite au passé de la subjectivité pour achever le récit de son aventure d'écrivain par la scène de la page blanche.

Pourtant dans la toute dernière phrase, une sorte de commentaire justificatif, l'écrivain revient au présent. "Dans toute cette histoire incompréhensible, ce qui reste le plus incompréhensible c'est le fait que le maitre d'école qui ce jour-là disparut dans le bois, on ne le revit jamais ; non, on ne retrouva pas même son cadavre ». Par cet « envoi ", Schnitzler, d'une chiquenaude, réintroduit le fantastique comme ferment de l'absurde. Il prend le lecteur à partie : l'univers de la fiction et son univers de lecteur ne font qu'un. Il lui fait l'universelle prédiction : dans les bois erre la mort à grande écharpe verte. Elle traversera, demain peut-être, la scène où il joue son rôle, sa vie.

$43 \mathrm{Au}$ terme de cette lecture, il convient de rendre justice à Freud... et à Schnitzler. L'univers de Schnitzler n'est pas celui de Poe. Structures, procédés et thèmes voisins font partie du fonds commun à toute écriture fantastique; du monde de revenants et d'énigmes scientifiques de Poe se dégage une atmosphère toute différente de celle de Schnitzler. Une analyse de leurs pessimismes, non-conformismes et ironies respectifs rapprocheraient sans doute davantage Schnitzler et Poe... Freud pour sa part met en évidence une des ambiguïtés du texte de Schnitzler, qui se révèle bien dépasser la simple intrigue romanesque.

La poétique du fantastique mise en œuvre dans La prédiction permet à Schnitzler une approche particulière de la peur de vivre, «la peur de la causalitée ${ }^{14}$ ", ici élevée au rang de combat singulier avec la mort. Le héros de Schnitzler, projetant sa perception sur le monde, le rend inquiétant. L'inquiétante étrangeté n'est pas celle des événements, elle est au cœur du héros. Ailleurs dans l'œuvre de Schnitzler d'autres techniques littéraires - appel à l'hypnose, monologue intérieur, jeu dans le jeu... - rendent sensible cette singularité de la perception. Quelques années plus tard Kafka fera de chaque perception singulière du monde - y compris celles dites fantastiques - la donnée première de ses œuvres, rendant le recours aux procédés littéraires fantastiques traditionnels en partie caducs. 

dupe, sans doute, des limites de ce genre, dont abusent trop souvent, à son gré, les chevaliers d'industrie de la littérature. "S'occuper d'occultisme devrait être interdit à tous ceux qui n'ont pas une connaissance suffisante de ce qui est - relativement patent; on ne devrait pas reconnaitre le droit de se mouvoir dans le territoire de l'inconscient à qui conque n'a pas d'abord parcouru en tous sens celui du conscient, scrupuleusement et jusqu'aux frontières de l'obscur. Mais bien entendu c'est justement dans ces régions aux frontières incertaines qu'aventuriers, spéculateurs et chevaliers d'industrie de la pensée sont le plus à l'aise !15 ".

Ce scrupuleux observateur et créateur souverain n'est-il pas Schnitzler lui-même, cherchant inlassablement des chemins dans le vaste territoire de l'âme, explorant ses frontières, découvrant ses clairs-obscurs?

\section{NOTES}

1. Arthur Schnitzler, "Die Weissagung», dans Gesammelte Werke, Die Erzählenden Schriften, S. Fischer Verlag, 1961, I, pp. 598-619.

Ce récit fut rédigé, en partant d'un plan ancien qui portait le titre Hexerei, en juin et juillet 1902. Première publication dans le numéro spécial de Noël de la Neue Freie Presse (Wien, 24.12.1905, Weihnachtsbeilage, S. 31-38). Première édition dans le troisième recueil de nouvelles de Schnitzler, intitulé Dämmerseelen, S. Fischer Verlag, 1907 (Das Schicksal des Freiherrn von Leisebogh Die Weissagung - Das neue Lied - Die Fremde - Andreas Thameyers letzter Briel).

2. Sigmund Freud, « Das Unheimliche» (1919), dans Studienausgabe, Bd. IV, Fischer Taschenbuch Verlag, 1982, p. 273.

3. Voir note 1. À propos de ce titre Dominique Auclères rapporte cette réflexion de Schnitzler âgé : «J'ai trouvé un beau titre: La Pénombre des Ames, mais il date de ma jeunesse. Il y a des moments maintenant où il m'accable » (Dans Arthur Schnitzler, Les dernières cartes, Calman-Lévy, 1953, p. XV).

4. Lettre du 17 avril 1907 à S. Fischer, dans Arthur Schnitzler, Briefe 1875-1912, Fischer, 1981, pp. 557-558: «meine Novellen: Variationen über Themen aus E. Poes Gedankenwelt [...] sonderbare Bemerkung [...] oberflächliche Betrachtungsweise».

5. En 1889 Schnitzler publia une étude «Über funktionelle Aphonie und deren Behandlung durch Hypnose und Suggestion» dans la Internationale Klinische Rundschau.

6. Voir à ce sujet l'autobiographie de Schnitzler, Jugend in Wien, dtv, 1971. Années 87-88, pp. 284-285.

7. Tzvetan Todorov, Introduction à la littérature fantastique, Ed. du Seuil, 1970, p. 176.

8. Voir l'analyse d'Irène Bessiere, dans Le récit fantastique, Larousse, 1974, pp. 229-232.

9. Voir supra, note 1.

10. Idem.

11. Op. cit., p. 608.

12. "Der Geist im Wort und der Geist in der Tat», dans Arthur Schnitzler, Aphorismen und Betrachtungen, S. Fischer Verlag, 1963, p. 150.

13. «Schicksal und Wille», dans Aphorismen, op. cit., p. 40. 
14. «Das Grauen gegenüber der Kausalität kann höchst vielfältige Formen anneh men, von unklaren Bangigkeil- und Angstgefühlen bis zur eigentlichen Lebensangst, und es kann ästhetisch sublimiert werden bis zum Weltschmerz», "Wunder und Gesetze», dans Aphorismen, op. cit., p. 72.

15. Idem, p. 71.

\section{RÉSUMÉS}

L'article analyse comment la logique narrative et sémantique de ce texte de 1902 fait de La prédiction un récit fantastique exemplaire. Il montre parallèlement que cette poétique du fantastique, à laquelle Schnitzler fit rarement appel, est ici une technique permettant une approche singulière de thèmes constants dans son œuvre (Sein / Schei - Spiel / Leben - Traum / Leben - Lüge / Wirklichkeit), et de leur connotation avec Je thème de l'âme crépusculaire (Dämmnerseelen « Âmes crépusculaires » - est le titre du recueil où figure La prédiction).

Le recours au fantastique met en évidence les symptômes de la non-adaptation à la vie d'un individu sans réelle force vitale et mal à l'aise dans l'univers social, mental et moral, qui, impressionné par la vision de sa mort suggérée par un magicien, travaille méthodiquement durant dix ans selon le schéma de cette vision - en fonction d'un délire morbide inavoué - à la réaliser. Le thème de la mort prédite, prévue, qu'une réalisation fictive - théâtrale - ne peut exorciser, renvoie au thème de la vision et du discours, au thème de la vacuité de l'art, dès lors qu'il est incapable de créer un univers authentique.

Texte littéraire fantastique, La prédiction marque une limite du genre dans la mesure où l'inquiétante étrangeté siège dans l'être humain et non dans le désordre du monde.

Der Artikel analysiert, wie die narrative und seinantische Logik dieses Textes von 1902 aus der Weissagung eine exemplarische phantastische Erzählung macht. Parallel dazu zeigt er, dass dicse Poetik des Phant astischen, auf die sich Schnitzler nur selten beru fen hat, hier eine Technik darstellt, die eine einzigarlige Annäherung an die immer wiederkehrenden Themen in seinen Werken (Sein / Schein - Spiel / Leben - Traum / Leben - Lüge / Wirklichkeit) und deren Konnotation des Motivs der Dämmerseele ermöglicht. Die Zuhilfenahme des Phantastischen verdeutlicht die Symptome der Unangepasstheit eines Menschen an das Leben, eines Menschen, der ohne cchte Lebenskraft und mit Unbehagen iri der sozialen, geistigen und moralischen Umwelt lebt, und der, unter dem Eindruck der Vision vom eigenen Tod, die ihm von einem Zauberer suggeriert wurde, systematisch und aufgrund cines uneingestandenen Todeswahns, zehn Jahre Jang an der Verwirklichung eben dieser Vision arbeitet. Das Motiv des vorausgesagten und vorausgesehenen Todes, den eine fiktive Aufführung nicht verhindern kann, vcrweist auf das Motiv der Leere der Kunst, wenn sie sich als unfähig erweist, ein authentisches Universum zu erschaffen. Als literarischer phantastischer Text macht Die Weissagung die Grenzen dieses Genres deutlich, insofern ais das Unheimliche nicht im Chaos der Welt, sondern im Menschen selbst zu suchen ist. 
AUTEUR

GENEVIĖVE ROUSSEL

Université de Lille III 